\title{
Toxoplasmose em primatas neotropicais: estudo retrospectivo de sete $\operatorname{casos}^{1}$
}

\author{
Renata A. Casagrande ${ }^{2 *}$, Tiffany C.E. da Silva ${ }^{2}$, Caroline A. Pescador ${ }^{3}$, Vanessa \\ Borelli ${ }^{2}$, Júlio C. Souza Jr ${ }^{4}$, Edson R. Souza ${ }^{5}$ e Sandra D. Traverso ${ }^{2}$
}

\begin{abstract}
Casagrande R.A., Silva T.C.E., Pescador C.A., Borelli V., Souza Jr J.C., Souza E.R. \& Traverso S.D. 2013. [Toxoplasmosis in New World primates: Retrospective study of seven cases.] Toxoplasmose em primatas neotropicais: estudo retrospectivo de sete casos. Pesquisa Veterinária Brasileira 33(1):94-98. Laboratório de Patologia Animal, Centro de Ciências Agroveterinárias, Universidade do Estado de Santa Catarina, Lages, SC 88520-000, Brazil. E-mail: casagrande_vet@yahoo.com.br

Toxoplasmosis is considered to be a fatal parasitic disease in New World primates. The objective of this report was to describe, through a retrospective study, the toxoplasmosis cases in New World primates. From 1999 to 2009 eighty-six anatomopathological exams was performed in primates and toxoplasmosis was the most common disease (7/86). One case occurred in Black-Tufted-Marmoset (Callithrix penicillata) and six in Brown-Howler monkeys (Alouatta guariba). Two monkeys were found death and five died within few days. The most common clinical signs were apathy and anorexia (5/7), abdominal distension $(4 / 7)$ and fever (3/7). The main gross lesions were splenomegaly $(4 / 7)$, hemorrhage in digestory system, lymph nodes and urinary bladder (4/7), reddish lungs (3/7) and hepatomegaly $(2 / 7)$. Histopathological findings were necrotizing hepatitis $(7 / 7)$, splenitis (3/7), myocarditis (2/7), enteritis (2/7), lymphadenitis (1/7) and sialitis (1/7), and interstitial pneumonia (4/7). In the liver, lungs, spleen, heart, lymph nodes and salivary gland there were tachyzoites of Toxoplasma gondii that also detected by immunohistochemistry against T. gondii in liver, spleen and lungs (5/7). Toxoplasmosis can cause high mortality in New World primate colonies and can be a threat to preservation of those species in captivity. Therefore, preventive measures should be taken to avoid the contamination of those animals.
\end{abstract}

INDEX TERMS: Toxoplasma gondii, toxoplasmosis, pathology, Neotropical primates, Santa Catarina.

RESUMO.- A toxoplasmose é considerada uma doença parasitária fatal em primatas neotropicais. 0 objetivo deste trabalho foi descrever, através de um estudo retrospectivo, os casos de toxoplasmose em primatas neotropicais. No

\footnotetext{
${ }^{1}$ Recebido em 11 de maio de 2012.

Aceito para publicação em 4 de setembro de 2012.

${ }^{2}$ Laboratório de Patologia Animal, Centro de Ciências Agroveterinárias, Universidade do Estado de Santa Catarina (CAV/UDESC), Av. Luiz de Camões 2090, Bairro Conta Dinheiro, Lages, SC 88520-000, Brasil. Autor para correspondência: casagrande_vet@yahoo.com.br

${ }^{3}$ Laboratório de Patologia Veterinária, Faculdade de Agronomia e Medicina Veterinária, Universidade Federal do Mato Grosso (UFMT), Av. Fernando Corrêa da Costa 2367, Bairro Boa Esperança, Cuiabá, MT 78069-900, Brasil.

${ }^{4}$ Centro de Pesquisas Biológicas de Indaial, Universidade de Blumenau (FURB), Rua Rio de Janeiro 401, Bairro Estados, Indaial, SC 89130-000, Brasil.

${ }^{5}$ Parque Zoobotânico de Brusque, Rua Manoel Tavares s/n, Brusque, SC 88350-450, Brasil.
}

período de 1999-2009 foram realizados 86 exames anatomopatológicos em primatas e a toxoplasmose foi a enfermidade mais comum (7/86), relatando-se um caso em sagui-do-tufo-preto (Callithrix penicillata) e seis em bugio-ruivo (Alouatta guariba). Dois animais foram encontrados mortos e cinco morreram em poucos dias. Os sinais clínicos mais frequentes foram apatia e anorexia (5/7), distensão abdominal (4/7) e febre (3/7). Na necropsia observou-se esplenomegalia (4/7), hemorragia do trato digestório, linfonodos e bexiga (4/7), pulmões avermelhados (3/7) e hepatomegalia $(2 / 7)$. No exame histopatológico evidenciou-se hepatite $(7 / 7)$, esplenite $(3 / 7)$, miocardite $(2 / 7)$, enterite (2/7), linfadenite (1/7) e sialite (1/7) necróticas e, pneumonia intersticial (4/7). Em fígado, pulmões, baço, coração, linfonodos e glândula salivar havia taquizoítos de Toxoplasma gondii que foram também detectados pelo exame de imuno-histoquímica anti-T. gondii em fígado, baço e pul- 
mões (5/7). A toxoplasmose pode causar alta mortalidade em colônias de primatas neotropicais e representar mais uma ameaça à conversação dessas espécies em cativeiro. Sendo assim, medidas preventivas devem ser tomadas para evitar a contaminação desses animais.

TERMOS DE INDEXAÇÃO: Toxoplasma gondii, toxoplasmose, patologia, primatas neotropicais, Santa Catarina.

\section{INTRODUÇÃO}

A toxoplasmose é uma enfermidade infecciosa cosmopolita, causada pelo protozoário intracelular obrigatório Toxoplasma gondii, que afeta uma grande variedade de mamíferos e aves (Jones et al. 2000).

0 gato doméstico e os felídeos selvagens são os hospedeiros definitivos, nos quais o parasito faz um ciclo enteroepitelial com excreção de oocistos junto às fezes no meio ambiente (Dubey et al. 1998). Nos hospedeiros intermediários, como os primatas, a reprodução do parasito se dá de forma assexuada, em todo o organismo, produzindo cistos teciduais (Dubey et al. 1998, Jones et al. 2000).

A transmissão desse agente pode ocorrer mediante ingestão das suas três formas infectantes: Ingestão de oocistos esporulados, por qualquer grupo animal, devido a contaminação fecal de alimentos e água; ingestão de taquizoítos, bradizoítos e/ou cistos teciduais pelos carnívoros (Dubey et al. 1998) e pela infecção transplacentária em hospedeiros intermediários (Dubey et al. 1995, Jones et al. 2000).

A toxoplasmose apresenta-se nas formas clínicas aguda ou crônica (Jones et al. 2000), as quais dependem basicamente da interação parasita-hospedeiro (Innes 1997, Dubey et al. 1998). Do ponto de vista do parasito, fatores como, tipo de cepa envolvida e a sua patogenicidade estão relacionados com o curso da doença. Quanto ao hospedeiro, o estado imunológico e a espécie infectada são os fatores mais importantes (Innes 1997, Dubey et al. 1998, Epiphanio et al. 2003).

Nos casos agudos, os órgãos mais afetados são os pulmões, fígado, baço, linfonodos, intestino e cérebro, cujas alterações são secundárias à necrose tecidual resultante da replicação e ruptura das células hospedeiras pelos taquizoítos (Jones et al. 2000). Quando o hospedeiro adquire resistência, a infecção torna-se crônica e nestes casos são visíveis cistos de bradizoítos, em locais como cérebro, musculatura esquelética e cardíaca (Jones et al. 2000, Dubey 2004).

Dentre os animais selvagens, a toxoplasmose é responsável por um grande incremento na mortalidade de primatas não humanos em cativeiro e em populações de vida livre, já que estes comumente desenvolvem infecção aguda e fatal. São vários os surtos relatados em colônias de primatas não humanos pelo mundo (Hessler et al. 1971, Borst \& Van Knapen 1984, Cunningham et al. 1992, Dietz et al. 1997, Inoue 1997, Pertz et al. 1997, Dubey et al. 2006) e em zoológicos e criadouros do Brasil (Bouer et al. 1999, Epiphanio et al. 2003, Andrade et al. 2007, Maluenda et al. 2009).

As espécies neotropicais são consideradas altamente susceptíveis a toxoplasmose, havendo relatos que apontam que esses animais são mais vulneráveis que os primatas do Velho Mundo (Anderson \& McClure 1993, Innes 1997, Silva 2007). Entretanto Anderson \& McClure (1993), Innes (1997), Epiphanio et al. (2003) referem que essa vulnerabilidade ainda não está bem esclarecida. Uma das hipóteses é que durante a evolução, os primatas neotropicais estiveram isolados dos felídeos, devido ao hábito arborícola e, portanto, do contato com oocistos de T. gondii, tornando-se mais sensíveis à doença (Innes 1997).

0 objetivo deste trabalho foi descrever, através de um estudo retrospectivo, os casos de toxoplasmose em primatas neotropicais diagnosticados no Laboratório de Patologia Animal (LAPA) do Centro de Ciências Agroveterinárias da Universidade do Estado de Santa Catarina (CAV/ UDESC), no período de 1999 a 2009.

\section{MATERIAL E MÉTODOS}

Um estudo retrospectivo das doenças diagnosticadas através de exames anatomopatológicos em primatas neotropicais pelo LAPA (CAV/UDESC) foi efetuado abrangendo o período de 1999 a 2009. As amostras com diagnóstico de toxoplasmose foram reprocessadas rotineiramente para exame histológico e coradas pela técnica Hematoxilina-Eosina (HE). Os dados clínicos presentes nos históricos foram agrupados e compilados.

Adicionalmente, fragmentos de pulmão, fígado e baço de todos os casos de toxoplasmose foram submetidos à técnica de imuno-histoquímica (IHQ). Após a desparafinização, as lâminas foram submetidas ao bloqueio da peroxidase endógena com peróxido de hidrogênio a 3\%. Na recuperação antigênica foi utilizado tripsina a $0,1 \%$ a $37^{\circ} \mathrm{C}$, e posterior irradiação em forno microondas de uso doméstico com imersão em tampão citrato (pH 6,0). Utilizou-se anticorpo policlonal anti-Toxoplasma gondii na diluição de 1:1000 a temperatura de $37^{\circ} \mathrm{C}$ por 1 hora. A IHQ foi realizada pela técnica de estreptavidina-biotina-peroxidade (Kit LSAB, Dako Cytomation, EUA). A revelação foi procedida com o cromógeno diaminobenzidina (Dako) e contracorados com hematoxilina. Como controle positivo foi utilizado fragmentos de encéfalo de um canino com cinomose que apresentava coinfecção por T. gondii.

\section{RESULTADOS}

No período de 1999-2009 foram realizados 86 exames anatomopatógicos em primatas neotropicais. Todos estes animais eram provenientes de diferentes municípios do Estado de Santa Catarina. Quanto à espécie, 44 eram bugios-ruivo (Alouatta guariba), sendo 32 de cativeiro, sete de vida livre e cinco de origem não informada; um macaco-aranha-preto (Ateles paniscus); seis macacos-prego (Sapajus apella); um mico-leão-cara-dourada (Leontopithecus chrysomelas); dois saguis-cara-branca (Callithrix geofroy); dois saguis-tufo-branco (Callithrix jacchus); 17 saguis-tufo-preto (Callithrix penicillata) e 13 saguis (Callithrix spp.), todos estes de cativeiros (zoológicos, criadouros ou domicílios).

Dos 86 exames efetuados nos primatas neotropicais, $30,2 \%$ (26/86) não tiveram diagnóstico conclusivo, ou por insuficiência de material ou por estado adiantado de autólise. Dos casos conclusivos, 25,6\% (22/86) morreram por apresentarem doença infecciosa ou parasitária; $24,4 \%$ (21/86) doenças degenerativas com destaque para a insuficiência renal crônica; 11,6\% (10/86) devido a distúrbios 
causados por agentes físicos; 3,5\% (3/86) por neoplasia; $3,5 \%(3 / 86)$ doença nutricional e 1,2\% (1/86) devido à intoxicação fitógena.

A enfermidade infecciosa mais prevalente foi a toxoplasmose, perfazendo um total de 11,6\% (7/60) de todos os diagnósticos conclusivos, sendo que dentre as doenças infecciosas ou parasitárias essa doença teve prevalência de $31,8 \%(7 / 22)$.

A primeira descrição de toxoplasmose ocorreu em 2006 em uma colônia de sagui-do-tufo-preto (Callithrix penicillata) do município de Lages. Estes animais viviam soltos em uma residência e em três dias, cinco animais foram encontrados mortos sem sinais clínicos prévios e um desses foi enviado para necropsia (caso 1). 0 segundo relato ocorreu no mesmo ano, em um parque Zoobotânico, em dois bugio-ruivos (Alouatta guariba) (casos 2 e 3), mantidos cativos juntos, com circulação de felinos domésticos

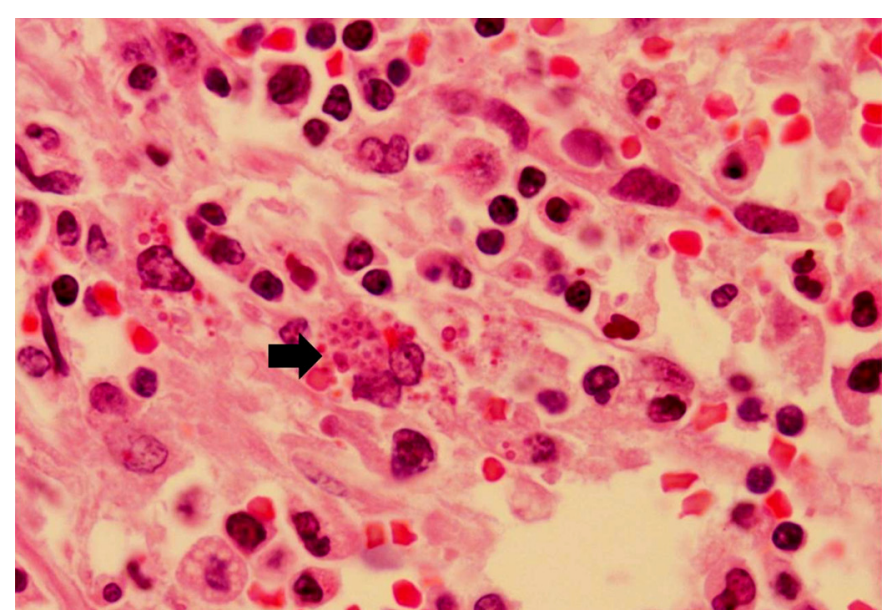

Fig.1. Toxoplasmose em Bugio-ruivo (Alouatta guariba). Baço com infiltrado de histiócitos e taquizoíto de Toxoplasma gondii no citoplasma de macrófago (seta). HE, obj.100x.

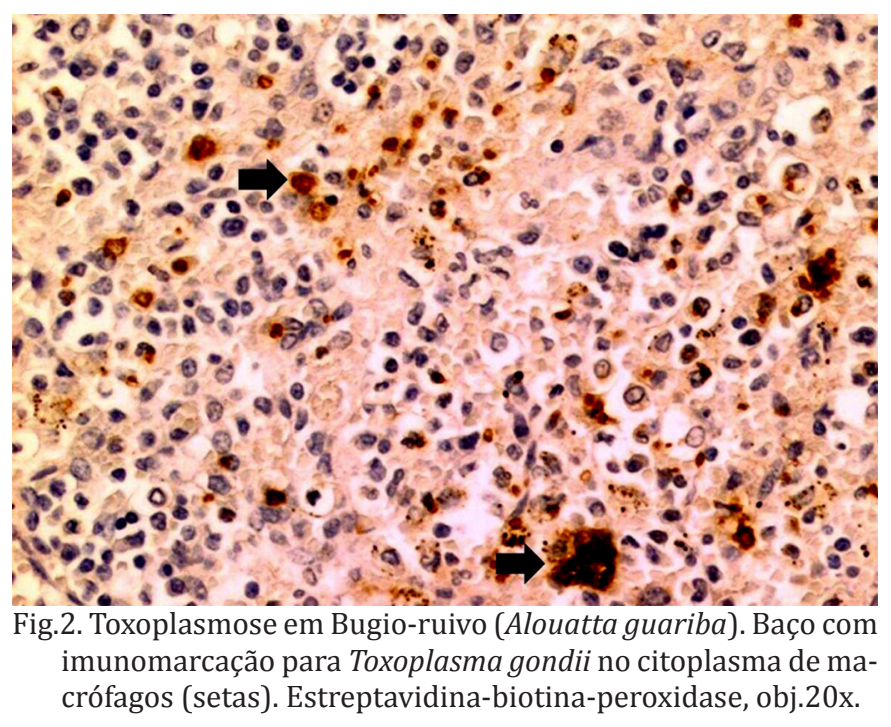

no interior do recinto. 0 terceiro relato foi diagnostico em 2006 em um bugio-ruivo de um Centro de Pesquisas Biológicas que vivia em semi-cativeiro (caso 4) e num intervalo de um ano, mais três animais contactantes também morreram de toxoplasmose (casos 5, 6 e 7). Todas as mortes ocorrem em poucos dias após o início dos sinais clínicos. As manifestações clínicas, achados de necropsia e histopatológicos dos casos de toxoplasmose encontram-se no Quadro 1.

O diagnóstico de toxoplasmose foi realizado através das alterações anatomopatológicas, visualização de estruturas piriformes a ovaladas, basofílicas sugestivas de taquizoítos de Toxoplasma gondii livres ou no interior de macrófagos (Fig.1) intralesionais (Quadro 1) e IHQ anti-T. gondii. No exame de IHQ observou-se marcação no citoplasma de macrófagos ou livres em baço (Fig.2), fígado e pulmão (casos 1 a 5). Não houve imunomarcação nos casos 6 e 7.

Quadro 1. Manifestações clínicas, achados de necropsia e histopatológicos dos casos de toxoplasmose em primatas neotropicais

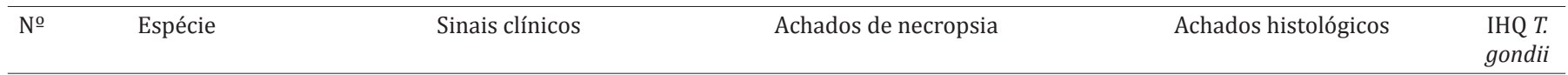

1 Sagui-do-tufo-preto (Cal- Encontrado morto lithrix penicillata)

2 Bugio-ruivo (Alouatta Apatia, anorexia, febre, dispneia, es- NI guariba)

3 Bugio-ruivo tertor pulmonar

Encontrado morto

NI

4 Bugio-ruivo

Apatia, anorexia, agressividade, ptialismo, distensão abdominal

Esplenomegalia; hemorragias em estômago, intestino e bexiga

5 Bugio-ruivo

Apatia, anorexia, febre, distensão abdominal, tosse improdutiva

6 Bugio-ruivo

7 Bugio-ruivo e firmes mões avermelhados nal tômago, intestino e bexiga
Pulmões avermelhados heterogêneos Hepatite*, esplenite* e miocardite* ne-

crótica multifocal moderada; Pneumonia* intersticial moderada

Hepatite* necrótica multifocal moderada; Pneumonia* intersticial discreta Hepatite* e esplenite* necrótica multifocal moderada; Pneumonia* intersticial moderada

Hepatite* necrótica multifocal moderada; enterite necro-hemorrágica segmentar moderada

Esplenomegalia; hepatomegalia; he- Hepatite*, esplenite* e linfadenite* nemorragias em estômago, intestino, crótica multifocal acentuada; Pneumo linfonodos mesentéricos e bexiga; Pul- nia* intersticial moderada

Apatia, anorexia, distensão abdomi- Esplenomegalia; hemorragias em es-

Apatia, anorexia, febre, mucosas pá- Esplenomegalia; hepatomegalia, helidas, tosse improdutiva, distensão morragias em pulmões, estômago, abdominal e constipação intestinal intestino e bexiga; ascite;
Hepatite* necrótica multifocal moderada; enterite necro-hemorrágica segmentar moderada

Hepatite* e miocardite necrótica multimoderada focal moderada; sialite* necrótica focal

$\overline{\mathrm{NI}}=$ não informado. ${ }^{*}$ Órgãos com taquizoítos de Toxoplasma gondii. 


\section{DISCUSSÃO E CONCLUSÃO}

No presente estudo anatomopatológico retrospectivo em primatas neotropicais, a toxoplasmose foi a enfermidade mais prevalente. Todos esses animais com toxoplasmose foram encontrados mortos ou morreram de forma aguda com sinais clínicos inespecíficos. Esses achados são comuns em primatas neotropicais com essa doença o que dificulta o diagnóstico ante mortem e a tentativa de tratamento (Cunningham et al. 1992, Anderson \& McClure 1993, Bouer et al. 1999, Epiphanio et al. 2003, Verona \& Pissinatti 2007, Maluenda et al 2009).

0 curso da doença é variável dentre as espécies de primatas neotropicais. Os saguis (Callithrix sp.) são extremamente susceptíveis a doença e podem morrer dentro de 111 dias após o contato inicial com o agente (Potkay 1992). Em um surto da doença em macacos-de-cheiro (Saimiri sciureus), os animais morreram em um período de quatro semanas (Borst \& Van Knapen 1984) e micos-leões-dourados (Leontopithecus rosalia rosalia) em um zoológico morreram poucas horas após serem observados clinicamente sadios (Pertz et al. 1997).

Nos sete casos analisados, os achados macro e microscópicos observados foram semelhantes com aqueles descritos na toxoplasmose em primatas e compreenderam, principalmente, alterações necróticas em fígado, baço, linfonodos mesentéricos, pulmão e coração, com lesões variadas ou ausentes no trato gastrointestinal (Hessler et al. 1971, Cunningham et al. 1992, Dietz et al. 1997, Inoue 1997, Bouer et al. 1999, Epiphanio et al. 2003, Dubey et al. 2006, Maluenda et al. 2009). Lesões encefálicas são descritas (Jones et al. 2000, Epiphanio et al. 2003), no entanto nos casos desse estudo não foram encontradas lesões macroscópicas e histológicas. Em um bugio-ruivo havia sialite necrótica focal moderada associada à taquizoítos de T. gondii, lesão essa ainda não reportada.

Dos sete casos analisados, em todos se evidenciou taquizoítos de T. gondii livres ou no interior de macrófagos intralesionais. A presença de formas parasitárias sejam elas taquizoítos ou cistos de bradizoítos são visualizados nos cortes histológicos, principalmente nas áreas com lesão, mas também sem associação direta com o infiltrado inflamatório (Hessler et al. 1971, Cunningham et al. 1992, Dietz et al. 1997, Inoue 1997, Bouer et al. 1999, Epiphanio et al. 2003, Dubey et al. 2006). O diagnóstico definitivo de toxoplasmose nem sempre é possível por avaliação anatomopatológica, uma vez que os parasitas podem não ser visualizados e quando da presença dos mesmos estes devem ser diferenciados de outros membros da família Sarcocystidae, como por exemplo, Neospora caninum (Jones et al. 2000).

Esta enfermidade em primatas, bem como em outros mamíferos, pode ser confirmada pelo exame de IHQ (Cunningham et al. 1992, Inoue 1997, Epiphanio et al. 2003, Dubey et al. 2006, Andrade et al. 2007, Maluenda et al. 2009). Dos setes casos avaliados no presente estudo, em cinco houve a confirmação através desse exame. Nos dois casos em que a IHQ foi negativa, o material permaneceu por longo período em formol. A fixação dos tecidos com este fixador pode levar a alterações estruturais nas proteínas (antí- genos) presentes na amostra (Ramos-Vara \& Beissenherz 2000). Segundo Webster et al. (2009), a fixação em formol por até 7 semanas não interfere na reação, no entanto, alguns anticorpos perdem a capacidade de ligar-se aos antígenos da amostra após longo período de fixação.

No presente trabalho não se pode comprovar a origem da infecção por T. gondii, pois os saguis (caso 1) viviam soltos e os bugio-ruivos estavam sendo mantido em semi-cativeiro (casos 4 ao 7). É possível que os bugios dos casos 2 e 3 tenham entrado em contato direto com o parasito, tendo em vista o histórico de circulação de felinos domésticos no interior do recinto onde os mesmos eram mantidos cativos.

A via de transmissão da toxoplasmose para primatas mantidos em cativeiro pode ocorrer pelo contato direto com o oocisto presente nas fezes de felídeos, tanto do próprio zoológico (felídeos selvagens) como por animais sinantrópicos (gatos domésticos) (Hessler et al. 1971 Dietz et al. 1997, Inoue 1997, Bouer et al. 1999, Dubey et al. 2006) ou atribuída ao consumo de carne fresca ou alimentos contaminados por cistos ou oocistos esporulados (Cunningham et al. 1992, Dietz et al. 1997, Inoue 1997, Bouer et al. 1999, Dubey et al. 2006). Neste sentido, é importante a construção de recintos de primatas afastados dos de felídeos e também o treinamento adequado dos tratadores para evitar a contaminação cruzada.

\section{CONCLUSÃO}

Através dos resultados obtidos nesse trabalho, conclui-se que a toxoplasmose é uma doença de curso clínico agudo e inespecífico com lesões histológicas características em diversos órgãos. Essa enfermidade pode causar alta mortalidade em colônias de primatas neotropicais e representar mais uma ameaça à conversação dessas espécies em cativeiro. Sendo assim, medidas preventivas devem ser tomadas para evitar a contaminação desses animais.

\section{REFERÊNCIAS}

Anderson D.C. \& McClure H.M. 1993. Toxoplasmosis, p.63-69. In: Jones T.C., Mohr U. \& Hunt R.D. (Eds), Monographs on Pathology of Laboratory Animals. I. Nonhuman primates. Springer-Verlag, New York. 234p.

Andrade M.C.R., Coelho J.M.C.O., Amendoeira M.R.R., Vicente R.T., Cardoso C.V.P., Ferreira P.C.B. \& Marchevsky R.S. 2007. Toxoplasmosis in squirrel monkeys: Histological and immunohistochemical analysis. Ciência Rural 37(6):1724-1727.

Borst G.H.A. \& Van Knapen F. 1984. Acute acquired toxoplasmosis in primates in a zoo. J. Zoo Anim. Med. 15(2):60-62.

Bouer A., Werther K., Catão-Dias J.L. \& Nunes A.L. 1999. Outbreak of toxoplasmosis in Lagothrix lagotricha. Folia Primatol. 70(5):282-285.

Cunningham A.A., Buxton D. \& Thomson K.M. 1992. An epidemic of toxoplasmosis in a captive colony of squirrel monkeys (Saimiri sciureus). J. Comp. Pathol. 107:207-219.

Dietz H.H., Henriksen P., Bille-Hansen V. \& Henriksen S.A. 1997. Toxoplasmosis in a colony of New World monkeys. Vet. Parasitol. 68:299-304.

Dubey J.P., Lappin M.R. \& Thulliez P. 1995. Diagnosis of induced toxoplasmosis in neonatal cats. J. Am. Vet. Med. Assoc. 207(2):179-185.

Dubey J.P., Lindsay D.S. \& Speer C.A. 1998. Structures of Toxoplasma gondii tachyzoites, bradyzoites, and sporozoites and biology and development of tissue cysts. J. Clin. Microbiol. 11(2):267-299.

Dubey J.P. 2002. A review of toxoplasmosis in wild birds. Vet. Parasitol. 106:121-153. 
Dubey J.P. 2004. Toxoplasmosis, a waterborne zoonosis. Vet. Parasitol. 126:57-72.

Dubey J.P., Hodgin E.C. \& Hamir A.N. 2006. Acute fatal toxoplasmosis in squirrels (Sciurus carolensis) with bradyzoites in visceral tissues. J. Parasitol. 92(3):658-659.

Epiphanio S., Sinhorini I.L. \& Catão-Dias J.L. 2003. Pathology of toxoplasmosis in captive New World primates. J. Comp. Pathol. 129:196204.

Hessler J.R., Woodard J.C. \& Tucek P.C. 1971. Lethal toxoplasmosis in a woolly monkey. J. Am. Vet. Med. Assoc. 159(11):1588-1594.

Innes E.A. 1997. Toxoplasmosis: comparative species susceptibility and host immune response. Comp. Immunol. Microbiol. Infect. Dis. 20(2): 131-138.

Inoue M. 1997. Acute toxoplasmosis in squirrel monkeys. J. Vet. Med. Sci. 59(7):593-595.

Jones T.C., Hunt R.D. \& King N.W. 2000. Patologia Veterinária. Manole, São Paulo. 1415p.

Maluenda A.C.H., Casagrande R.A., Nemer V.C., Kanamura C.T., Teixeira R.H.F. \& Matushima E.R. 2009. Infecção aguda fatal por Toxoplasma gondii em macaco barrigudo (Lagothrix lagotricha). Clín. Vet. 81:100104.
Pertz C., Dubelzig R.R. \& Linday D.S. 1997. Fatal Toxoplasma gondii infection in golden lion tamarins (Leontopithecus rosalia rosalia). J. Zoo Wildl. Med. 28:491-493.

Potkay S. 1992. Diseases of the Callitrichidae: A review. J. Med. Primatol. 21:189-236.

Ramos-Vara J.A. \& Beissenherz M.E. 2000. Optimization of immunohistochemical methods using two different antigen retrieval methods on formalin-fixed, paraffin-embedded tissues: experience with 63 markers. J. Vet. Diagn. Invest. 12:307-311.

Silva J.C.R. 2007. Toxoplasmose, p.768-784. In: Cubas Z.S., Silva J.C.R. \& Catão-Dias J.L. (Eds), Tratado de Animais Selvagens: medicina veterinária. Roca, São Paulo. 1354p.

Tenter A.M., Heckeroth A.R. \& Weiss L.M. 2000. Toxoplasma gondii: from animals to humans. Int. J. Parasitol. 30:1217-1258.

Verona C.E.S. \& Pissinatti A. 2007. Primates: primatas do novo mundo (sagui, macaco-prego, macaco-aranha, bugio), p.358-377. In: Cubas Z.S., Silva J.C.R. \& Catão-Dias J.L. (Eds), Tratado de Animais Selvagens: medicina veterinária. Roca, São Paulo. 1354p.

Webster J.D., Miller M.A., DuSold D. \& Ramos-Vara J.A. 2009. Effects of prolonged formalin fixation on diagnostic immunohistochemistry in domestic animals. J. Histochem. Cytochem. 57:753-761. 